(C) The Author(s), 2022. Published by Cambridge University Press on behalf of The Nutrition Society. This is an Open Access article, distributed under the terms of the Creative Commons Attribution licence (http://creativecommons.org/licenses/by/4.0/), which permits unrestricted re-use, distribution and reproduction, provided the original article is properly cited.

\title{
Degree of food processing and breast cancer risk in black urban women from Soweto, South African: the South African Breast Cancer study
}

Inarie Jacobs $^{1 *}$, Christine Taljaard-Krugell ${ }^{1}$, Mariaan Wicks ${ }^{1}$, Herbert Cubasch ${ }^{2,3}$, Maureen Joffe ${ }^{3,4}$, Ria Laubscher ${ }^{5}$, Isabelle Romieu ${ }^{6,7}$, Renata B. Levy ${ }^{8,9}$, Fernanda Rauber $^{8,9}$, Carine Biessy ${ }^{10}$, Sabina Rinaldi ${ }^{10}$ and Inge Huybrechts ${ }^{10}$

${ }^{1}$ Centre of Excellence for Nutrition, North-West University, Private Bag X6001, Potchefstroom 2520, South Africa

${ }^{2}$ Department of Surgery, Faculty of Health Sciences, University of Witwatersrand, Private Bag X2600, Houghton, Johannesburg 2041, South Africa

${ }^{3}$ Non-Communicable Diseases Research Division, Wits Health Consortium (PTY) Ltd, Parktown, Johannesburg 2193, South Africa ${ }^{4}$ MRC Developmental Pathways to Health Research Unit, Department of Paediatrics, Faculty of Health Sciences, University of Witwatersrand, Private Bag X3, Johannesburg 2050, South Africa

${ }^{5}$ South African Medical Research Council, PO Box 19070, Tygerberg, Cape Town, 7505 South Africa

${ }^{6}$ Centro de Investigación en Salud Poblacional, Instituto Nacional de Salud Pública, CP 62100, Cuernavaca, Morelos, México ${ }^{7}$ Hubert Department of Global Health, Emory University, Atlanta, GA 30329, USA

${ }^{8}$ Department of Preventive Medicine, School of Medicine, University of São Paulo (FMUSP), São Paulo, SP 01246-903, Brazil ${ }^{9}$ Center for Epidemiological Studies in Health and Nutrition, University of São Paulo (Nupens/USP), São Paulo, SP 01246-904, Brazil ${ }^{10}$ International Agency for Research on Cancer, Nutrition and Metabolism Branch, IARC-WHO 150 cours Albert Thomas, 69372 Lyon, France

(Submitted 6 August 2021 - Final revision received 14 January 2022 - Accepted 31 January 2022 - First published online 3 February 2022)

\section{Abstract}

This study aimed to investigate the association between consumption of ultra-processed foods, whole foods and breast cancer risk in black women from Soweto, South Africa. A population-based case ( $n$ 396)-control ( $n$ 396) study matched on age and residence, using data from the South African Breast Cancer study. Dietary intake was assessed using a validated quantified FFQ. Food items were categorised using the NOVA system ((1) unprocessed/minimally processed foods, (2) culinary ingredients, (3) processed foods and (4) ultra-processed foods). Conditional logistic regression models were used to estimate OR and $95 \% \mathrm{CI}$ of dietary contributions from each NOVA food group (as a percentage of total energy intake (EI)) and adjusting for potential confounders. Considering contributions to total EI per day, ultra-processed food consumption contributed to $44.8 \%$ in cases and $47.9 \%$ in controls, while unprocessed/minimally processed foods contributed to $38.8 \%$ in cases and $35.2 \%$ in controls. Unprocessed/minimally processed food consumption showed an inverse association with breast cancer risk overall $(\mathrm{OR}=0.52,95 \% \mathrm{CI} 0.35,0.78)$, as well as in pre- and postmenopausal women separately (OR $=0.52,95 \% \mathrm{CI} 0.27,0.95$ and $\mathrm{OR}=0.55$, $95 \% \mathrm{CI} 0 \cdot 35,0 \cdot 89$, respectively) and in women with progesterone positive breast cancer (OR $=0.23,95 \% \mathrm{CI} 0 \cdot 06,0 \cdot 86)$. There was no heterogeneity in association with breast cancer when analyses were stratified according to BMI. No significant associations were observed for the consumption of other NOVA food groups. Intake of unprocessed/minimally processed foods may reduce the risk of developing breast cancer in black women from Soweto, South Africa.

Key words: NOVA: Ultra-processed: Minimally/unprocessed foods: Breast cancer risk: South Africa

Breast cancer is currently the cancer with the highest incidence rate among South African women ${ }^{(1,2)}$. Modifiable risk factors such as an unhealthy diet, obesity and physical inactivity have already been identified and play a crucial role in primary breast cancer prevention ${ }^{(3)}$.
South Africa has several policies in line with the comprehensive NOURISHING (not an acronym, but a mnemonic for food policy action) policy framework of the World Cancer Research Fund and American Institute for Cancer Research, promoting

Abbreviations: EI, energy intake; PR+, progesterone positive; QFFQ, quantified FFQ; SABC, South African Breast Cancer.

* Corresponding author: Inarie Jacobs, email inarie.jacobs@gmail.com 
healthy diets and lifestyle behaviour that may reduce the risk of developing breast cancer ${ }^{(4)}$. However, as a result of the nutrition transition, the South African food environment is becoming more obesogenic where whole foods are frequently being replaced by ultra-processed foods ${ }^{(5,6)}$. According to the NOVA food processing classification system (not an acronym but a name and hereafter referred to as the NOVA system), ultra-processed foods are defined as 'formulations of ingredients, mostly of exclusive industry use, that result from a series of industrial processes ${ }^{(7)}$. Ultra-processed foods are becoming more accessible, available and affordable in both rural and urban areas of South Africa ${ }^{(8,9)}$. This is worrisome since ultra-processed food consumption has been associated with an increased risk of gaining weight or becoming obese and developing non-communicable diseases, including breast cancer ${ }^{(10-14)}$.

Not all aspects of food processing such as grinding, milling, freezing and drying of fresh foods are nutritionally harmful and some are mainly used to manufacture various nutritious staple foods (maize meal, frozen vegetables, etc. ${ }^{(7)}$. However, diets characterised by more frequent consumption of ultra-processed foods are more energy dense and contain less micro-nutrients and fibre than diets characterised with frequent consumption of unprocessed/minimally processed foods ${ }^{(14-16)}$.

Various classification systems have been proposed for categorising foods according to their degree of processing such as the International Food Information Council and University of North Carolina UNC systems, Foodex and LanguaL ${ }^{(17-21)}$. But, the NOVA system established by Monteiro and associates is used the most in research and policy development ${ }^{(7,21,22)}$. The NOVA system assigns foods to one of four food classification groups according to the extent and purpose of processing ${ }^{(21)}$. These groups include (1) unprocessed or minimally processed foods (fresh and frozen fruit and vegetables, meat, milk, etc.); (2) culinary ingredients (salt, sugar, oil and butter); (3) processed foods (smoked or cured meats, cheese, simple bread, foods preserved in salt, water or oil, etc.) and (4) ultra-processed foods (packed sweets, ready-to-eat foods, breakfast cereals, sauces, mass-produced breads, margarine, biscuits, etc.).

A previous study conducted by Jacobs and colleagues investigated the association between a posteriori dietary patterns and breast cancer risk in black urban South African women from Soweto. In this study, several patterns have been identified: a traditional pattern (composed of poultry, organ and offal meat, mono- and polyunsaturated fats, soup powders and vegetables), cereal-dairy breakfast-pattern (composed of milk, plain yogurt, unsweetened breakfast cereals, sorghum porridge and fruit juice, while being negatively correlated with maize meal porridge and saturated fats) and a processed food dietary pattern (composed of cheese, sweetened dairy products, candy/sugar, fast foods, alcoholic beverages, sugar-sweetened beverages, fruit spreads and crackers/potato crisps). While the traditionaland cereal-dairy breakfast dietary patterns were significantly inversely associated with breast cancer risk, no association was observed between a processed food dietary pattern and breast cancer risk ${ }^{(23)}$. However, this approach did not consider the degree of industrial food processing according to the NOVA system specifically. In addition, individual consumption data have never been assessed according to the degree of food processing in any South African study. Therefore, the aim of the present study was to (1) describe dietary intake of ultra-processed food according to the degree of food processing, using the NOVA system, in an urban population of Black South African women from Soweto, South Africa who participated in a population-based case-control study and (2) to investigate the association of UPF with breast cancer risk.

\section{Methods \\ Study population}

Data from the South African Breast Cancer (SABC) study, a population-based, case-control study among black urban women, were used for this study ${ }^{(24-26)}$. The SABC study was conducted at the Chris Hani Baragwanath Academic Hospital in Soweto from 2014 to 2017 and included a total of 399 cases and 399 matched controls. Due to missing dietary intake information, three cases and three controls were excluded. The final population of the study included 396 cases and 396 matched controls. Breast cancer cases (premenopausal $n$ 133; postmenopausal $n$ 248) included black urban adult women (over 18 years of age) who were newly diagnosed with breast cancer and who did not previously receive any cancer treatment. Breast cancer cases were invited to participate at referral for diagnostic biopsy, and failing that, as soon as possible after cancer diagnosis. Stage at breast cancer was clinically assessed at diagnoses and coded according to the tumour-node-metastasis classification. Breast cancer subtypes were defined using the Allred score ${ }^{(24)}$. Controls (premenopausal $n$ 134; postmenopausal $n$ 257) were black adult women from Soweto (over 18 years of age) and unrelated to breast cancer cases with no history of cancer diagnoses. Controls were recruited using a multistep sampling procedure from the areas of residency of the cases and matched by area of residence and age $\left( \pm 5\right.$ years ${ }^{(25,26)}$. Other matching criteria of interest (e.g. reproductive factors, weight and physical activity) were captured and used in statistical models to allow us to study these criteria in relation to breast cancer risk. The sample size (396 cases and 396 matched controls) was sufficient to obtain a power $>80 \%$ (type-II error rate $\beta=10 \%$ ) for $\mathrm{OR}_{\mathrm{s}} \geq 1.5$ when type-I error is set to $5 \%$. For subcategory analysis, a size of $\geq 132$ participants were sufficient to obtain a power $>80 \%$ (type-II error rate $\beta=10 \%$ ) for $\mathrm{OR}_{s} \geq 1.7$ when type-I error is set to $5 \%{ }^{(27)}$.

\section{Ethical approval}

The International Agency for Research on Cancer and the University of the Witwatersrand Committee for Research on Human Subjects granted ethical approval for the SABC study (M140980). The Gauteng Province Medical Advisory Committee gave permission to conduct research at the Chris Hani Baragwanath Academic Hospital. The North-West University gave ethical approval for the use of the quantified FFQ (QFFQ). All participants gave written informed consent prior to participation to the study. 


\section{General patient information and health assessments}

Trained investigators and fieldworkers conducted face-to-face interviews at inclusion in the study and for cases as soon as possible after breast cancer diagnoses. Information regarding socioeconomic and demographics (income, education and other household amenities) was self-reported. Detailed information was further collected with a questionnaire regarding history of health, ethnicity, reproductive risk factors, breast health, family history of cancer, physical activity and smoking habits. Anthropometric measurements such as height, sitting height, weight and waist circumference were performed according to a standardised protocol. BMI was calculated as $\mathrm{kg} / \mathrm{m}^{2}$. Questionnaires used to obtain the above-mentioned information were validated ${ }^{(28-30)}$.

\section{Dietary intake assessment}

Participants were asked about their habitual dietary intake over the past month (to reduce recall bias) and dietary intake data were collected immediately after breast cancer diagnoses (at recruitment) before any cancer treatment. A validated and reproducible culture-specific QFFQ was used together with food models, food portion pictures and household utensils alongside the South African Food Composition Tables to determine habitual dietary intake ${ }^{(31-33)}$. The QFFQ entailed of 145 food items reported by recently published literature as regularly consumed staple foods and foods less regularly consumed. The dietary intake frequency included the amount of times foods were consumed per day/week/month or never. Life size colour photographs of thirty-seven foods (in three portion sizes) were displayed in the food portion picture booklet ${ }^{(32)}$. A detailed description of the method used to determine the daily intakes is described elsewhere ${ }^{(34)}$. The nutrient and energy intakes (EI) were calculated by multiplying the daily intake of each food item by the nutrient and energy content (per $100 \mathrm{~g}$ ), derived from the South African Food Composition Tables, and then adding the contribution from all food items together ${ }^{(35)}$.

\section{Classifying foods based upon their level of processing}

To classify foods based on the level of food processing, all foods and drinks, collected through the QFFQ, were categorised into one of the four NOVA food groups. The NOVA system was used in our study since it is the most used food classification system in research studies and will enhance comparability of our study results $^{(21)}$. Foods were categorised into their most usual form of consumption with the most conservative classification option (less processed level) chosen in a case of doubt. Homemade dishes and culinary ingredients used in preparation methods (onions fried in oil or pumpkin candied with added sugar) were disaggregated according to the standardised recipes of the South African Food Composition Tables ${ }^{(35)}$. Disaggregated food items were then categorised based on the NOVA system. For instance, onions fried in oil were categorised as follows: onions as unprocessed/minimally processed food and oil as culinary ingredients.

Unprocessed or minimally processed foods are defined as foods that are naturally ready to consume or foods altered by minimal processing such as removal of inedible parts, drying, grinding, crushing, filtering, roasting, freezing, chilling, pasteurising, fermenting and boiling. These processing methods do not add any salt, sugar, oils or fats to the original products ${ }^{(7)}$. These foods included staple foods mostly consumed in South Africa (such as fresh fruits, $100 \%$ fruit juices, fresh and frozen vegetable, legumes, rice, maize meal, pasta, fresh milk, milk powder, eggs, meat and fish, homemade dishes such as stews and soups).

Culinary ingredients (NOVA 2) are substances extracted from unprocessed/minimally processed foods or from nature, such as salt, sugar, fat and oil ${ }^{(7)}$. Industrial processes, such as pressing, refining, centrifuging, mining or extracting, are mainly used in food preparation, seasoning and cooking of unprocessed/minimally processed foods ${ }^{(7)}$.

Processed foods (NOVA 3) are defined as industrial products made with added salt, sugar or other culinary ingredients to unprocessed/minimally processed foods, using preservation methods. For instance, bottling or canning and using non-alcoholic fermentation in the case of cheeses and breads ${ }^{(7)}$. The aim of food processing in this group is to increase the durability of foods within the unprocessed/minimally processed food group and to make them more enjoyable by enhancing their sensory qualities. Foods is this category include: cheese, preserved fruits, vegetables and beans in brine, salted nuts or nut spreads, sweetened dairy products (contains only added sugar with no additives), beer, wine and non-ultra-processed bakery products.

Ultra-processed foods (NOVA 4) included carbonated drinks, industrial pre-packaged fortified* bread and buns, cookies, pastries, cakes, cake mixes, breakfast cereals, energy bars, margarines and spreads, instants sauces, soup powders, pre-prepared pies, pasta and pizza dishes, reconstituted meat products, sweet and savoury packaged snacks, ice-cream, fruit yogurts, instant desserts and noodles and distilled alcohol products such as whiskey, gin, rum and vodka. All breads were considered to be industrial pre-packed breads and were classified under ultra-processed foods since the QFFQ used to determine dietary intake did not specify between processed or ultra-processed bread. *Fortification of wheat flour, used to make bread, is compulsory in South Africa since bread is considered a staple food (fortified with vitamin A, riboflavin, niacin, pyridoxine, thiamine, folic acid, Fe and $\mathrm{Zn}$ ).

\section{Statistical analysis}

Descriptive analyses were presented by mean values and standard deviations (data which were normally distributed) and median; 25th-75th percentile (for data with skewed distributions). Differences between breast cancer cases and controls were assessed using paired sample $t$ test (normal distributed data) and Wilcoxon signed rank test (skewed data) for continuous variables and paired $\chi^{2}$ test for categorical variables (presented as percentages).

For each participant, the relative energy contribution (percentage $\mathrm{kJ}$ of total EI/d) of each NOVA food group in the diet was calculated and then categorised into tertiles. Conditional logistic regression was applied to compute OR and associated $95 \%$ CI to determine the associations with each NOVA food group (dose-response analysis of 
tertiles) and breast cancer risk and when stratified by oestrogen positive and progesterone positive (PR+) receptors. Due to the limited number of participants in each breast cancer subcategory, which may have insufficient statistical power, we decided to explore associations by oestrogen positive and PR+ receptors only, and not in combination. Unconditional logistic regression was used to determine the association with breast cancer risk when analysis was stratified by menopausal status (pre $v$. post) and BMI status (BMI $<30 \mathrm{~kg}$ ) $\mathrm{m}^{2} v$. BMI $\geq 30 \mathrm{~kg} / \mathrm{m}^{2}$ ).

A two-stage model was used to obtain OR and the associated $95 \%$ CI. Confounding factors were considered as factors influencing the crude OR output by more than $10 \%$. The crude output was reported in model 1, taken into account the matched factors of breast cancer cases and controls (age, demographic factors). The following confounders were examined in the analysis before insertion in model 2: age (continuous), ethnicity (Zulu/Pedi/ Swazi, Xhosa, Sotho, Tshwane, Venda, Tsonga and Ndebele), individual income/month in South African Rand (zero income, income between R1-R3000 ( \pm \$0.1-\$195) and income $\geq$ R3001+ ( $> \pm \$ 195)$, categorised based on data from this study)', level of education (none/primary school, high school and college/postgraduate/diploma), smoking (smokers and non-smokers), height (continuous), waist circumference (continuous), total physical activity/d ( $\leq 16 \mathrm{~min} / \mathrm{d} v .>16 \mathrm{~min} / \mathrm{d}$, categorised based on median of current population), age at menarche (continuous), full-term pregnancy (yes/no), age at first pregnancy ( $\leq 24 v .>24$ years of age, categorised based on median age of the current population), age of menopause ( $\leq 48 v$. $>48$ years of age, categorised based on median age of the current population), parity ( $\leq 3$ children $v .>3$ children, categorised based on median number of children of the current population), ever breast-feeding (yes/no), duration of exclusive breast-feeding (months), use of exogenous hormones (hormonal birth control to avoid pregnancy such as oral contraceptives and injections or hormone replacement therapy/combined hormone replacement therapy after menopause), family history of breast cancer (yes/no), alcohol consumption, HIV positivity (yes/no) and reporting of energy (under-reporting $v$. over-reporting)

Only ethnicity, individual income per month, waist circumference (not adjusted for waist circumference when analysis was stratified by obesity status), physical activity and menopausal status (not adjusted for menopausal status when analysis was stratified by menopausal status) influenced the crude output by more than $10 \%$ and were therefore included in model 2.

A generalised linear model was used to estimate the differences in least square means (measured in $\mathrm{kJ}$ per day) of (1) unprocessed/minimally processed foods, (2) processed foods and (3) ultra-processed foods between breast cancer cases and control participants. The effect of potential confounders (the same potential confounders used in the regression model) was tested for by including additional variables into the generalised linear regression model.

\section{Results}

Table 1 presents the distribution of selected characteristics between breast cancer cases and control participants. Ethnicity differed significantly among cases and control participants with cases having more Ndebele-speaking people and controls having more Sotho-speaking people. Breast cancer cases had a significant lower waist circumference $(93.3 \mathrm{~cm}$ (sD 13.8) $\mathrm{cm}$ ) compared with controls $(95.8 \mathrm{~cm}(\mathrm{sD} 13.7) \mathrm{cm})$ and had a lower percentage of HIV-positive women (16.5\% v. 22.6\%). Considering dietary factors, the percentage of non-alcohol consumers was higher in cases $(80.8 \%)$ than in control participants (69.4\%). Additionally, in breast cancer case participants, oestrogen positive $(75.2 \%)$ and $\mathrm{PR}+(75.2 \%)$ were the dominant hormonal breast cancer tumour receptors, while triple negative breast cancer receptors accounted for $16 \cdot 1 \%$ of all tumour types.

With regard to total EI per day (of habitual dietary intake), ultraprocessed food consumption contributed to $44.8 \%$ in breast cancer cases and $47.9 \%$ in control participants $(P<0.05)$ (see Fig. 1). Unprocessed/minimally processed foods contributed to $38.8 \%$ in breast cancer cases and $35.2 \%$ in control participants, while processed food consumption contributed to $10.3 \%$ in breast cancer cases and $11.4 \%$ in control participants' total EI per day. Culinary ingredients contributed the least to total $\mathrm{EI} / \mathrm{d}$ in both breast cancer cases (6.1\%) and controls (5.5\%).

Table 2 provides the OR and $95 \%$ CI of unprocessed/minimally processed and ultra-processed foods in association with breast cancer risk. Trend analysis of the crude model showed that higher consumption of unprocessed/minimally processed foods was inversely associated with breast cancer risk overall (OR $=0.50,95 \%$ CI $0.34,0.73, P$-trend $<0.001)$, for postmenopausal women (OR $=0.50,95 \% \mathrm{CI} 0.32,0.78, P=0.003)$, for $\mathrm{PR}+$ breast cancers $(\mathrm{OR}=0.30,95 \% \mathrm{CI} 0.09,0.93, P$-trend $=0.037)$ and for obese women $(\mathrm{OR}=0.55,95 \%$ CI 0.35, 0.89, $P$-trend $=0 \cdot 014)$. Trend analysis further showed that higher consumption of culinary ingredients, processed foods and ultraprocessed foods was inversely associated with breast cancer risk overall $\quad(\mathrm{OR}=0.53,95 \%$ CI $0.36, \quad 0.75, \quad P$-trend $<0.001$, $\mathrm{OR}=0.53,95 \%$ CI $0.37,0.77, P$-trend $=0.001$ and $\mathrm{OR}=0.47$, $95 \%$ CI $0 \cdot 31,0 \cdot 72, P$-trend $<0 \cdot 001$, respectively).

After adjusting for possible confounders, the associations between breast cancer risk and higher consumption of unprocessed/minimally processed foods remained significant for breast cancer risk overall $(\mathrm{OR}=0.52,95 \%$ CI $0.35,0.78, P$-trend $=0.001)$, for postmenopausal women $(\mathrm{OR}=0.55$, 95 \% CI $0 \cdot 35,0 \cdot 89, P$-trend $=0 \cdot 012)$ for $\mathrm{PR}+$ breast cancers $(\mathrm{OR}=0 \cdot 23$, $95 \%$ CI $0.06,0.86, P$-trend $=0.029)$, for women with a $\mathrm{BMI}<30 \mathrm{~kg} / \mathrm{m}^{2}(\mathrm{OR}=0 \cdot 47,95 \%$ CI 0.26, 0.85, $P$-trend $=0 \cdot 014)$ and for women with a BMI $\geq 30 \mathrm{~kg} / \mathrm{m}^{2}(\mathrm{OR}=0 \cdot 57,95 \%$ CI $0 \cdot 35$, $0.92, P$-trend $=0.021)$. An inverse association with breast cancer risk and higher consumption of unprocessed/minimally processed foods was additionally noted for premenopausal women $(\mathrm{OR}=0.51,95 \%$ CI 0.27, 0.95, $P$-trend $=0.035)$.

No significant associations were observed between higher consumption of culinary ingredients, processed- or ultra-processed foods and breast cancer risk when adjusted for possible confounders. Sensitivity analysis was done by classifying industrial pre-packed bread under the processed food group (NOVA 3) (instead of under ultra-processed foods) in the analysis, since the QFFQ used to determine dietary intake did not specify whether breads consumed were processed or ultra-processed. However, sensitivity analysis did not significantly change the 
Table 1. Distribution of selected characteristics between cases and control participants (Mean values and standard deviations, percentiles based on distribution of variables and percentages)

\begin{tabular}{|c|c|c|c|c|c|}
\hline \multirow[b]{2}{*}{ Characteristics } & \multicolumn{2}{|c|}{ Controls (n 396) } & \multicolumn{2}{|c|}{ Cases (n 396) } & \multirow[b]{2}{*}{$P$} \\
\hline & $n$ & $\%$ & $n$ & $\%$ & \\
\hline
\end{tabular}

graphic factors

Age (years)

Mean

SD

$54 \cdot 6$

Ethnicity

Zulu/Pedi/Xhosa/Tswana/Swazi

Sotho

Venda/Tsonga

Ndebele

Level of education

None/primary

High school

College/University/postgraduate

Individual income/month

Ro

$\mathrm{R} 1-\mathrm{R} 3000(n / \%)$

$\mathrm{R}^{3} 001^{+}(n / \%)$

Anthropometry

BMI

Underweight $<18.5 \mathrm{~kg} / \mathrm{m}^{2}$

Normal weight $\geq 18.5$ and $\leq 24.9 \mathrm{~kg} / \mathrm{m}^{2}$

Overweight $\geq 25.0$ and $\leq 29.9 \mathrm{~kg} / \mathrm{m}^{2}$

Obese $\geq 30.0 \mathrm{~kg} / \mathrm{m}^{2}$

WC $(\mathrm{cm})$

Mean

SD

Lifestyle factors

Total vigorous and moderate PA min/week

Median

25th percentile, 75th percentile

Current smokers

HIV positivity

Dietary factors

$\mathrm{TE}(\mathrm{kJ} / \mathrm{d})$

25th percentile, 75 th percentile

Protein $(\mathrm{g} / \mathrm{d})$

Median

25th percentile, 75 th percentile

$\%$ of TE

Fat $(g / d)$

Median

25th percentile, 75th percentile

$\%$ of TE

$\mathrm{CHO}(\mathrm{g} / \mathrm{d})$

Mean

SD

$\%$ of TE

Dietary Fibre $(\mathrm{g} / \mathrm{d})$

Mean

SD

Added sugar $(\mathrm{g} / \mathrm{d})$

Median

25th percentile, 75th percentile

$\%$ of TE

Non-alcohol consumers

Ethanol intake (g/d)

Median

25th percentile, 75th percentile

NOVA food groups

Unprocessed/minimally processed foods $(\mathrm{g} / \mathrm{d})$

Median

25th percentile, 75 th percentile

Culinary ingredients $(\mathrm{g} / \mathrm{d})$

Median

25th percentile, 75 th percentile

\section{9}

66

144

91

95

71

279

46

108

227

61

5

63

93

235

95.8

13.7

$32 \cdot 1$

$9 \cdot 1,70 \cdot 8$

44

90

990

7184,10284

63.5

$49 \cdot 2,93 \cdot 1$

$12 \cdot 0$

64.4

$47 \cdot 2,95 \cdot 7$

$27 \cdot 2$

$338 \cdot 7$

$147 \cdot 3$

$64 \cdot 0$

$25 \cdot 4$

$11 \cdot 5$

67.9

$39.9,109.7$

12.0

275

4.6

$2.5 ; 14.7$

$1619 \cdot 4$

$1115 \cdot 2,2568 \cdot 0$

33.0

$16 \cdot 7,115 \cdot 0$
$54 \cdot 7$

12.9

0.980

0.041

$16 \cdot 6$

$36 \cdot 4$

23.0

24.0

67

108
105

105
116

$17 \cdot 9$

70.5

11.6

$27 \cdot 3$

64.2

8.5

$1 \cdot 3$

$15 \cdot 9$

23.5

$59 \cdot 3$

97

97
257

42

125

219

52

$16 \cdot 9$

$27 \cdot 3$

26.5

$29 \cdot 3$

24.5

64.9

10.6

$31 \cdot 6$

59.8

$8 \cdot 6$

$\begin{array}{rr}7 & 1.8 \\ 71 & 17.9\end{array}$

$87 \quad 21.9$

$231 \quad 58.4$

93.3

13.8

$39 \cdot 4$

$7 \cdot 9,85 \cdot 8$

35

65

22.6

9146

6812,9759

63.8

$47 \cdot 4,82 \cdot 7$

11.8

$64 \cdot 8$

$42 \cdot 4,91 \cdot 9$

26.9

330.8

143.5

$61 \cdot 4$

24.9

11.0

$65 \cdot 3$

$38.4,105.5$

$12 \cdot 1$

$69 \cdot 4$

320

$80 \cdot 8$

$<0.001$

$5 \cdot 4$

$2.8 ; 13.8$

$1480 \cdot 5$

$1060 \cdot 8,2032 \cdot 8$

$26 \cdot 5$

$14 \cdot 1,77 \cdot 5$

0.078

0.350

0.790

0.011

0.286

0.025

0.073

$0 \cdot 125$

0.445

0.616

0.313

0.005

0.029

0.081 
Table 1. (Continued)

\begin{tabular}{|c|c|c|c|c|c|}
\hline \multirow[b]{2}{*}{ Characteristics } & \multicolumn{2}{|c|}{ Controls ( $n$ 396) } & \multicolumn{2}{|c|}{ Cases ( $n$ 396) } & \multirow[b]{2}{*}{$P$} \\
\hline & $n$ & $\%$ & $n$ & $\%$ & \\
\hline \multicolumn{5}{|l|}{ Processed foods (g/d) } & \multirow[t]{3}{*}{$<0.001$} \\
\hline Median & \multirow{2}{*}{\multicolumn{2}{|c|}{$\begin{array}{l}87 \cdot 1 \\
28 \cdot 3,303 \cdot 0\end{array}$}} & \multicolumn{2}{|l|}{63.9} & \\
\hline 25th percentile, 75 th percentile & & & $18 \cdot 2$, & & \\
\hline \multicolumn{6}{|l|}{ Ultra-processed foods $(\mathrm{g} / \mathrm{d})$} \\
\hline Median & $753 \cdot 5$ & & $603 \cdot 9$ & & \multirow[t]{2}{*}{$<0.001$} \\
\hline 25th percentile, 75 th percentile & $340 \cdot 2$ & & $300 \cdot 1$ & & \\
\hline \multicolumn{6}{|l|}{ Breast cancer risk factors } \\
\hline Full term pregnancy in parous women & 382 & 100 & 377 & 100 & 0.374 \\
\hline Ever breastfed in parous women & 349 & $91 \cdot 3$ & 339 & 89.9 & 0.293 \\
\hline Duration of breast-feeding (1) (months) & 32 & 12,60 & 30 & 8,58 & 0.187 \\
\hline Use of birth control (contraceptives) & 215 & 54,3 & 229 & 57.8 & 0.316 \\
\hline Postmenopausal & 257 & 64.8 & 248 & $62 \cdot 6$ & 0.852 \\
\hline Age at menopause (2) (years) & 48 & 44,50 & 47 & 42,50 & 0.331 \\
\hline Family history of $\mathrm{BC}$ & 17 & $4 \cdot 3$ & 25 & $6 \cdot 3$ & 0.205 \\
\hline \multicolumn{6}{|l|}{ Age at menarche } \\
\hline 25th percentile, 75th percentile & 13,16 & & 13,16 & & 0.537 \\
\hline \multicolumn{6}{|l|}{ Breast cancer case characteristics } \\
\hline \multicolumn{6}{|l|}{ Stage at BC diagnoses } \\
\hline I & - & & 24 & 6.5 & \\
\hline II & - & & 175 & 44.8 & \\
\hline III & - & & 161 & $40 \cdot 8$ & \\
\hline IV & - & & 31 & 7.9 & \\
\hline \multicolumn{6}{|l|}{ Receptor status } \\
\hline $\mathrm{ER}+$ & - & & 298 & $75 \cdot 2$ & - \\
\hline $\mathrm{PR}+$ & - & & 263 & 66.4 & - \\
\hline HER2 & - & & 114 & $28 \cdot 8$ & - \\
\hline \multicolumn{6}{|l|}{ Breast cancer subtypet } \\
\hline HER2 enriched & - & & 21 & $5 \cdot 3$ & - \\
\hline Luminal $\mathrm{A}$ & - & & 40 & $10 \cdot 1$ & - \\
\hline Luminal B & - & & 269 & $67 \cdot 9$ & - \\
\hline TNBC & - & & 64 & $16 \cdot 2$ & - \\
\hline
\end{tabular}

WC, waist circumference; TE, total energy; CHO, carbohydrates; PA, physical activity; ER+, oestrogen receptor positive; PR+ progesterone receptor positive; HER2, human-epidermal growth factor-2; TNBC, triple negative breast cancer; HRT, hormone replacement therapy.

Continuous variables are presented as means and standard deviations if normally distributed and median (25th percentile, 75th percentile) if not, categorical variables are presented as percentages.

* 16 Missing values for menopausal status (15 cases and 1 control). Missing values were excluded from percentage calculations

$\dagger$ Defined using Allred score.

(1) In breast-feeding women only.

(2) Among postmenopausal women only.

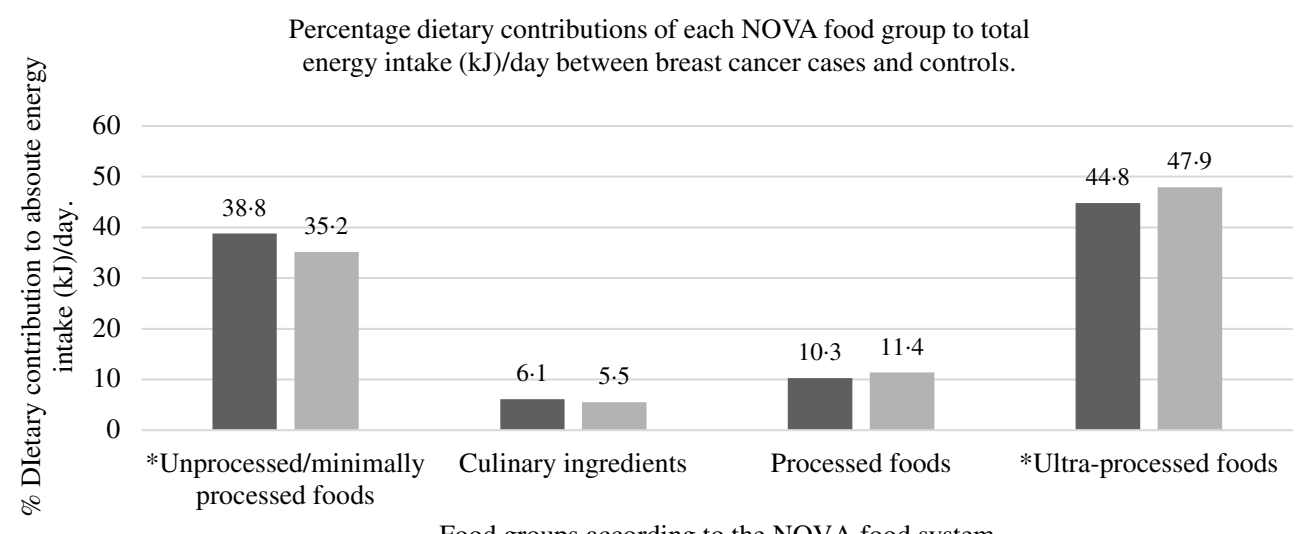

Food groups according to the NOVA food system

Fig. 1. Percentage dietary contributions of each NOVA food group to total energy intake (kJ) (per day) between $(\square)$ breast cancer cases and ( $\square$ ) controls.

results. Sensitivity analysis was conducted, using the proportion of each NOVA food group as a weighted ratio (percentage $\mathrm{g} / \mathrm{d}$ ) of the total food intakes to take into account non-nutritional factors that do not provide any energy (artificial sweeteners and additives) in ultra-processed foods. Results did not differ from the analysis with food groups measured as percentage $\mathrm{kJ} / \mathrm{d}$. 
Table 2. Relative intakes (percentage of total energy intake) for each NOVA food group (dose-response analysis of tertiles) in association with breast cancer risk (Odds ratios and $95 \%$ confidence intervals)

\begin{tabular}{|c|c|c|c|c|c|c|c|c|c|c|c|c|c|c|c|c|}
\hline & \multicolumn{4}{|c|}{$\begin{array}{l}\text { Unprocessed/minimally processed } \\
\text { foods }\end{array}$} & \multicolumn{4}{|c|}{ Culinary ingredients } & \multicolumn{4}{|c|}{ Processed foods } & \multicolumn{4}{|c|}{ Ultra-processed foods } \\
\hline & \multicolumn{2}{|c|}{ Model 1} & \multicolumn{2}{|c|}{ Model 2} & \multicolumn{2}{|c|}{ Model 1} & \multicolumn{2}{|c|}{ Model 2} & \multirow{2}{*}{$\begin{array}{c}\text { Model } \\
1 \\
\text { OR } \\
\end{array}$} & \multirow[b]{2}{*}{$95 \% \mathrm{Cl}$} & \multicolumn{2}{|c|}{ Model 2} & \multicolumn{2}{|c|}{ Model 1} & \multicolumn{2}{|c|}{ Model 2} \\
\hline & OR & $95 \% \mathrm{Cl}$ & OR & $95 \% \mathrm{Cl}$ & OR & $95 \% \mathrm{Cl}$ & OR & $95 \% \mathrm{Cl}$ & & & OR & $95 \% \mathrm{Cl}$ & OR & $95 \% \mathrm{Cl}$ & OR & $95 \% \mathrm{Cl}$ \\
\hline \multicolumn{17}{|c|}{ Overall (cases $n$ 396; controls ( $n$ 396) } \\
\hline Lowest tertile & 1 & & 1 & & 1 & & 1 & & 1 & & 1 & & 1 & & 1 & \\
\hline Middle tertile & 1.02 & $0.73,1.42$ & 1.01 & $0.72,1.43$ & 0.90 & $0.65,1.26$ & 0.84 & $0.59,1.19$ & 0.83 & $0.59,1.17$ & 0.79 & $0.54,1.14$ & 1.28 & $0.91,1.83$ & 1.36 & $0 \cdot 89,2 \cdot 10$ \\
\hline Highest tertile & 0.50 & $0.34,0.73$ & 0.52 & $0.35,0.78$ & 0.53 & $0.36,0.75$ & 0.55 & $0.38,1.32$ & 0.53 & $0.37,0.77$ & 0.86 & $0.63,1 \cdot 15$ & 0.47 & $0.31,0.72$ & 1.03 & $0.72,1.45$ \\
\hline \multirow{2}{*}{\multicolumn{17}{|c|}{$\begin{array}{l}\text { Premenopausal (cases } n \text { 148; controls } \\
n 139)^{\star}\end{array}$}} \\
\hline & & & & & & & & & & & & & & & & \\
\hline Lowest tertile & 1 & & 1 & & 1 & & 1 & & 1 & & 1 & & 1 & & 1 & \\
\hline Middle tertile & 0.72 & $0.42,1.26$ & 0.66 & $0 \cdot 37,1 \cdot 18$ & 1.00 & $0.58,1.72$ & 0.94 & $0.54,1.65$ & 0.68 & $0.39,1 \cdot 21$ & 0.74 & $0.41,1.32$ & $1 \cdot 14$ & $0.64,2 \cdot 05$ & 1.25 & $0 \cdot 68,2 \cdot 25$ \\
\hline Highest tertile & 0.56 & $0.31,1.03$ & 0.51 & $0.27,0.95$ & 0.74 & $0.41,1 \cdot 33$ & 0.67 & $0.37,1.24$ & 0.43 & $0.23,0.78$ & 0.45 & $0.24,1.56$ & 0.63 & $0.32,1 \cdot 22$ & 0.62 & $0 \cdot 32,1 \cdot 22$ \\
\hline$P$-trend & 0.062 & & 0.035 & & 0.311 & & 0.212 & & 0.192 & & 0.445 & & 0.171 & & 0.168 & \\
\hline \multicolumn{17}{|c|}{$\begin{array}{l}\text { Postmenopausal (cases } n 248 \text {; con- } \\
\text { trols } n \text { 257) }\end{array}$} \\
\hline Lowest tertile & 1 & & 1 & & 1 & & 1 & & 1 & & 1 & & 1 & & 1 & \\
\hline Middle tertile & 1.23 & $0.81,1.86$ & 1.26 & $0.83,1.92$ & 0.77 & $0.51,1 \cdot 18$ & 0.72 & $0.46,1 \cdot 10$ & 0.91 & $90.59,1.38$ & 0.90 & $0.59,1.38$ & 1.41 & $0.93,2 \cdot 12$ & 1.44 & $0.95,2 \cdot 12$ \\
\hline Highest tertile & 0.50 & $0.32,0.78$ & 0.55 & $0.35,0.89$ & 0.45 & $0 \cdot 29,1 \cdot 10$ & 0.46 & $0.29,1 \cdot 33$ & 0.60 & $0.39,1.23$ & 0.65 & $0.42,1.03$ & 0.48 & $0.30,0.75$ & 0.89 & $0.62,1 \cdot 29$ \\
\hline$P$-trend & 0.003 & & 0.012 & & 0.201 & & 0.112 & & 0.122 & & 0.070 & & 0.104 & & 0.130 & \\
\hline \multicolumn{17}{|c|}{$\mathrm{ER}+$ (cases $n$ 298) } \\
\hline Lowest tertile & 1 & & 1 & & 1 & & 1 & & 1 & & 1 & & 1 & & 1 & \\
\hline Middle tertile & 0.86 & $0 \cdot 32,2 \cdot 28$ & 0.91 & $0 \cdot 31,2 \cdot 71$ & 0.78 & $0.29,2.09$ & 1.03 & $0.34,3.13$ & $2 \cdot 13$ & $0.78,5 \cdot 83$ & 2.33 & $0.75,7.26$ & 3.69 & $1 \cdot 31,10 \cdot 41$ & $5 \cdot 41$ & $1.66,7.91$ \\
\hline Highest tertile & 0.52 & $0.21,1 \cdot 30$ & 0.53 & $0.19,1.48$ & 0.64 & $0.30,1.38$ & 0.75 & $0.32,1.73$ & 0.71 & $0.32,1.51$ & 0.79 & $0.34,1.82$ & 1.01 & $0.42,2 \cdot 45$ & 1.31 & $0.48,3.57$ \\
\hline$P$-trend & 0.165 & & 0.226 & & 0.260 & & 0.505 & & 0.369 & & 0.581 & & 0.974 & & 0.586 & \\
\hline \multicolumn{17}{|c|}{$\mathrm{PR}+($ cases $n$ 263) } \\
\hline Lowest tertile & 1 & & 1 & & 1 & & 1 & & 1 & & 1 & & 1 & & 1 & \\
\hline Middle tertile & 0.53 & $0 \cdot 16,1 \cdot 71$ & 0.52 & $0.14,1.97$ & 1.38 & $0 \cdot 45,4 \cdot 21$ & 1.61 & $0.42,0.62$ & 1.27 & $0.42,3.81$ & $2 \cdot 29$ & $0.56,0.93$ & 4.43 & $1 \cdot 29,6 \cdot 39$ & 8.95 & $1.93,41.45$ \\
\hline Highest tertile & 0.30 & $0.90,0.93$ & 0.23 & $0.06,0.86$ & 0.71 & $0.31,1.65$ & 0.74 & $0.28,1.94$ & 0.48 & $0.19,1 \cdot 19$ & 0.51 & $0.17,1.55$ & 0.89 & $0.33,2.44$ & 1.11 & $0.34,3.58$ \\
\hline \multirow{2}{*}{\multicolumn{17}{|c|}{$\begin{array}{l}\mathrm{BMl}<30 \mathrm{~kg} / \mathrm{m}^{2} \text { (cases = } 165 ; \text { controls } \\
\quad \quad 161)\end{array}$}} \\
\hline & & & & & & & & & & & & & & & & \\
\hline Lowest tertile & 1 & & 1 & & 1 & & 1 & & 1 & & 1 & & 1 & & 1 & \\
\hline Middle tertile & 1.15 & $0.68,1.93$ & 1.07 & $0.61,1.87$ & 1.31 & $0.77,2 \cdot 23$ & 1.32 & $0 \cdot 77,2 \cdot 27$ & 1.08 & $0.64,1 \cdot 82$ & 1.04 & $0.60,1.81$ & 1.25 & $0.75,2 \cdot 01$ & 1.22 & $0 \cdot 67,2 \cdot 25$ \\
\hline Highest tertile & 0.50 & $0.28,0.88$ & 0.47 & $0.26,0.85$ & 0.61 & $0.35,1.05$ & 0.58 & $0.33,1 \cdot 01$ & 1.21 & $0.84,1 \cdot 73$ & 0.50 & $0 \cdot 29,1 \cdot 23$ & $1 \cdot 18$ & $0 \cdot 82,1 \cdot 71$ & 1.01 & $0.59,1.52$ \\
\hline$P$-trend & 0.117 & & 0.014 & & 0.074 & & 0.056 & & 0.310 & & 0.872 & & 0.354 & & 0.516 & \\
\hline \multicolumn{17}{|c|}{$\begin{array}{l}\text { Obesity (cases } n 231 \text {; controls } n \text { 235) } \\
{ }^{*}, \dagger\end{array}$} \\
\hline Lowest tertile & 1 & & 1 & & 1 & & 1 & & 1 & & 1 & & 1 & & 1 & \\
\hline Middle tertile & 0.93 & $0.61,1.43$ & 0.89 & $0.57,1.37$ & 0.64 & $0.42,0.98$ & 0.61 & $0.39,0.94$ & 0.70 & $0.45,1.08$ & 0.70 & $0.45,1.09$ & 1.35 & $0.88,2 \cdot 08$ & 1.3 & $0.84,2.02$ \\
\hline Highest tertile & 0.55 & $0.35,0.89$ & 0.57 & $0.35,0.92$ & 0.48 & $0.31,1.04$ & 0.51 & $0.32,1 \cdot 21$ & 0.56 & $0.36,1.09$ & 0.59 & $0.37,1.03$ & 0.64 & $0.40,1.05$ & 0.67 & $0.41,1.08$ \\
\hline$P$-trend & 0.014 & & 0.021 & & 0.071 & & 0.203 & & 0.116 & & 0.115 & & 0.077 & & 0.103 & \\
\hline
\end{tabular}

$\mathrm{ER}+$, oestrogen receptor positive; $\mathrm{PR}+$, progesterone receptor positive

Model 1: crude output.

Model 2: adjusted for individual income per month, ethnicity, physical activity, waist circumference (not adjusted for waist circumference when stratified by obesity status) and menopausal status (not adjusted for menopause when stratified by menopausal status).

* Unconditional logistic regression.
† Obesity defined as $\mathrm{BMI} \geq 30 \mathrm{~kg} / \mathrm{m}^{2}$. 
Table 3 presents the nutrient profile of each NOVA food group (comparing the highest tertile of each NOVA food group). The unprocessed/minimally processed food group had the lowest total energy content (median $=7344 \mathrm{~kJ}, 5764-9172 \mathrm{~kJ}$ ) followed by culinary ingredients $($ median $=9619 \mathrm{~kJ}, 7484-12338$ $\mathrm{kJ}$ ), processed foods (median $=11314 \mathrm{~kJ}, 8334-14862 \mathrm{~kJ}$ ) and the ultra-processed food group showing the highest total energy content (median $=10 \quad 982 \mathrm{~kJ}, \quad 7561-14 \quad 958 \mathrm{~kJ})$. Comparing all the NOVA food groups, the ultra-processed food group had the highest content of saturated fat (median $=22.9 \mathrm{~g}$, $14.9-32.5 \mathrm{~g}$ ) and added sugar (median $=82.3 \mathrm{~g}, 41.8-141.9 \mathrm{~g}$ ) while having the lowest content of dietary fibre (mean $=19.7 \mathrm{~g}$ (sD 13.1) g). The unprocessed/minimally processed food group had the highest content of dietary fibre $($ mean $=28.1 \mathrm{~g}(\mathrm{sD} 14.9) \mathrm{g})$ while having the lowest content of saturated fats (median $=14.4 \mathrm{~g}$, $10 \cdot 1-21.5 \mathrm{~g}$ ) and added sugar (median $=60.8 \mathrm{~g}, 34.9-95.6 \mathrm{~g}$ ). Both the unprocessed/minimally processed and ultra-processed food groups had similar high contents of micronutrients.

Online Supplementary Table S1 presents the top ten single foods consumed between breast cancer cases and controls within the unprocessed/minimally processed, processed and ultra-processed food groups. The culinary ingredients group was excluded from this table. Maize meal, white rice, apples, eggs and beetroot were among the most consumed single foods within the unprocessed/minimally processed food group in both breast cancer cases and controls.

Regarding processed foods, peanut butter, atchar (sweet and/or spiced condiment usually made from chillies, vegetables or mangoes), vetkoek (deep fried doughnut like dough) scones and canned pilchards (small fatty fish, also referred to as sardines) were among the top ten single foods consumed in both breast cancer cases and controls.

The most consumed single foods within the ultra-processed food group in both breast cancer cases and control participants were brown bread, white bread, soup powder, beef sausages (minced meat with salt, spices and other additives), margarine and carbonated soft drinks.

\section{Discussion}

In this study of black women from Soweto, South Africa, consumption of unprocessed/minimally processed foods contributed to more than a third of the total EI per day in both case and control participants, while ultra-processed foods contributed to more than $44 \%$ and processed food to about $12 \%$ of the total EI per day. Higher consumption of unprocessed/minimally processed foods was inversely associated with breast cancer risk overall, for pre- and postmenopausal women, for $\mathrm{PR}+$ breast cancers, for women with a BMI $<30 \mathrm{~kg} / \mathrm{m}^{2}$ and for women with a BMI $\geq 30 \mathrm{~kg} / \mathrm{m}^{2}$. No significant association with breast cancer risk was observed for higher consumption of culinary ingredients, processed and ultra-processed foods.

\section{Consumption of unprocessed/minimally processed foods} and breast cancer risk

Unprocessed/minimally processed food consumption accounted for $38.8 \%$ of total EI in breast cancer cases and
$35.2 \%$ in controls in this study. Studies investigating the association between breast cancer risk and unprocessed/minimally processed foods (NOVA 1), as a group, are lacking. However, similar intakes of unprocessed/minimally processed foods were observed in studies (investigating the association between unprocessed/minimally processed foods and risk of developing other non-communicable diseases or being obese) conducted in Canada (39.2\%), Australia (35\%) and Chile (33.8\%) $15,36,37)$.

Results of our study are in line with other studies (conducted in different populations), which have found an inverse association between plant-based unprocessed/minimally processed food groups or dietary patterns with non-communicable diseases $^{(38,39)}$. In general, plant-based unprocessed/minimally processed foods have a healthier nutrient profile with a lower energy density and high content of various phytochemicals (i.e. fibre, carotenoids and flavonoids), vitamins and minerals, which may be linked to several anti-cancerous cell effects in the human body ${ }^{(3,37)}$. The unprocessed/minimally processed food group in our study had a similar 'healthy' nutrient profile as these plant-based unprocessed/minimally processed foods, which may explain the significant inverse association thereof with breast cancer risk in our study. Unprocessed or minimally processed foods are also linked to a lower risk of gaining weight or being obese, which may reduce the risk of developing postmenopausal breast cancer ${ }^{(3)}$. Disease prevention dietary guidelines such as the World Cancer Research fund/American Institute for Cancer Research's Cancer Prevention Recommendations and the South African Food Based Dietary Guidelines therefore advise to consume unprocessed whole foods (maize meal, fruits, vegetables, milk and lean meats) while limiting highly processed foods with added sugar, saturated fat and salt ${ }^{(3,40)}$. Results of this study support the use of the above-mentioned dietary guidelines as educational tools to promote healthy diets in Soweto, South Africa that could assist in preventing breast cancer development.

\section{Consumption of culinary ingredients and processed foods}

The contribution of culinary ingredients and processed foods to total EI/d was similarly distributed in both breast cancer cases (6.1 and $10.9 \%$, respectively) and controls (5.5 and $12.8 \%$, respectively). Similar percentage energy contributions of culinary and processed foods to total $\mathrm{EI} / \mathrm{d}$ were noted in other studies (Australia, UK, Canada and the USA) ${ }^{(15,37,41-43)}$. Higher consumption of culinary ingredients and processed foods (although very differently defined) did not show any significant association with breast cancer risk in our study. More research regarding culinary ingredients and processed food consumption is required in South Africa and in particular this population.

\section{Ultra-processed food consumption and breast cancer risk}

Dietary contributions of ultra-processed foods to total EI per day in this study (> 44\%) were lower than studies conducted in the USA (between 55.0 and $57.5 \%$ ) and the UK (between 48.6 and $56 \cdot 8 \%)^{(41-43)}$. Our study showed similar percentage intakes of ultra-processed foods as in studies conducted in Canada (48\%) and Australia (42\%), while lower percentages of ultraprocessed food consumption were noted in Belgium (29.6\%), France $(18.7 \%)$ and other middle-income countries such as 
NS British Journal of Nutrition

Table 3. Nutrient profile of each NOVA food group overall (comparing the highest tertile of each food group) (Median values and percentiles; mean values and standard deviations)

\begin{tabular}{|c|c|c|c|c|c|c|c|c|}
\hline & \multicolumn{2}{|c|}{$\begin{array}{l}\text { Unprocessed/minimally processed } \\
\text { foods }\end{array}$} & \multicolumn{2}{|c|}{ Culinary ingredients } & \multicolumn{2}{|c|}{ Processed foods } & \multicolumn{2}{|c|}{ Ultra-processed foods } \\
\hline & Median & 25th-75th percentiles & Median & 25th-75th percentiles & Median & 25th-75th percentiles & Median & 25th-75th percentiles \\
\hline Energy $(\mathrm{kJ})^{\star}$ & 7344 & 5764-9172 & 9619 & 7484-12 338 & 11314 & 8334-14 862 & 10982 & 7561-14958 \\
\hline Total protein $(\mathrm{g})^{\star}$ & 74.2 & $56.7-95.7$ & 62.6 & $47 \cdot 5-82 \cdot 4$ & 79.4 & $55 \cdot 4-105 \cdot 7$ & 72.5 & $52 \cdot 8-107 \cdot 2$ \\
\hline Plant protein $(\mathrm{g})^{\star}$ & 34.8 & $24 \cdot 9-48.5$ & 28.8 & $21 \cdot 8-37.9$ & $25 \cdot 1$ & $19 \cdot 7-31 \cdot 7$ & 31.8 & $23 \cdot 8-45 \cdot 3$ \\
\hline Animal protein $(g)^{\star}$ & $37 \cdot 4$ & $25 \cdot 2-52 \cdot 6$ & 31.5 & $21 \cdot 1-45 \cdot 6$ & $40 \cdot 1$ & $26 \cdot 4-56 \cdot 3$ & 38.0 & $23 \cdot 1-57 \cdot 3$ \\
\hline Total fat $(\mathrm{g})^{*}$ & 76.8 & $49 \cdot 9-103 \cdot 5$ & 71.4 & $49 \cdot 3-100 \cdot 9$ & 83.7 & $56 \cdot 5-110 \cdot 7$ & 77.9 & $50 \cdot 2-109 \cdot 4$ \\
\hline Saturated fat $(\mathrm{g})^{\star}$ & 14.4 & $10 \cdot 1-21 \cdot 5$ & $17 \cdot 1$ & $11 \cdot 3-25 \cdot 2$ & $22 \cdot 8$ & $16 \cdot 1-31 \cdot 5$ & 21.9 & $14.9-32.5$ \\
\hline Monounsaturated fat $(\mathrm{g})^{*}$ & 24.2 & $15 \cdot 9-32 \cdot 7$ & $22 \cdot 3$ & $14 \cdot 6-31 \cdot 7$ & $26 \cdot 5$ & $17 \cdot 9-35 \cdot 1$ & 24.4 & $15 \cdot 9-34.4$ \\
\hline Polyunsaturated fat $(\mathrm{g})^{\star}$ & $21 \cdot 1$ & $13.5-29 \cdot 8$ & $19 \cdot 8$ & $12 \cdot 8-29 \cdot 2$ & 23.7 & $15 \cdot 2-32 \cdot 6$ & 21.3 & $13 \cdot 7-30 \cdot 8$ \\
\hline Cholesterol (g) & 198.0 & $125 \cdot 7-282 \cdot 1$ & $252 \cdot 3$ & $151 \cdot 0-399 \cdot 1$ & 330.4 & $192 \cdot 9-490 \cdot 2$ & 286.8 & $166 \cdot 5-432 \cdot 3$ \\
\hline \multicolumn{9}{|l|}{ Total $\mathrm{CHO}(\mathrm{g}) \dagger$} \\
\hline Mean & 339.4 & & 328.6 & & 354.7 & & $346 \cdot 1$ & \\
\hline $\mathrm{SD}$ & $185 \cdot 2$ & & 177.4 & & 173.6 & & 179.9 & \\
\hline Added sugar $(\mathrm{g})^{\star}$ & 60.8 & $34.9-95.6$ & 84.1 & $43 \cdot 4-154.4$ & 79.2 & $41 \cdot 9-121 \cdot 1$ & $82 \cdot 3$ & $41.8-141.9$ \\
\hline \multicolumn{9}{|l|}{ Dietary fibre $(\mathrm{g}) \dagger$} \\
\hline Mean & 28.1 & & 21.4 & & $19 \cdot 8$ & & 19.7 & \\
\hline SD & 14.9 & & 10.9 & & 9.7 & & $13 \cdot 1$ & \\
\hline Protein: $\mathrm{CHO}$ : Fat ratio & $1: 4 \cdot 6: 2 \cdot 3$ & & $1: 5 \cdot 2: 2 \cdot 5$ & & $1: 4 \cdot 5: 2 \cdot 4$ & & $1: 4 \cdot 8: 2 \cdot 4$ & \\
\hline $\mathrm{Ca}(\mathrm{mg})^{\star}$ & $552 \cdot 1$ & $367 \cdot 0-815 \cdot 6$ & $404 \cdot 3$ & $244.7-621.3$ & 363.9 & $288 \cdot 1-543 \cdot 4$ & $520 \cdot 8$ & $365 \cdot 1-717 \cdot 2$ \\
\hline \multicolumn{9}{|l|}{$\mathrm{Fe}(\mathrm{mg}) \dagger$} \\
\hline Mean & $17 \cdot 2$ & & $15 \cdot 1$ & & $17 \cdot 2$ & & $17 \cdot 1$ & \\
\hline SD & 9.4 & & 6.7 & & 5.9 & & 8.9 & \\
\hline \multicolumn{9}{|l|}{$M g(\mu g) \dagger$} \\
\hline Mean & $352 \cdot 1$ & & 309.9 & & 319.7 & & 344.9 & \\
\hline SD & $195 \cdot 4$ & & 143.7 & & 119.1 & & 171.4 & \\
\hline$P(m g)^{\star}$ & $1139 \cdot 2$ & $816 \cdot 7-1679 \cdot 8$ & 946.7 & $704.7-1227.5$ & 1068.8 & $815 \cdot 7-1331 \cdot 1$ & 1121.8 & $899.9-1437.5$ \\
\hline $\mathrm{K}(\mathrm{mg})^{\star}$ & 2770.5 & $1998 \cdot 1-3724 \cdot 1$ & $2086 \cdot 3$ & $1562 \cdot 1-2784 \cdot 3$ & $1956 \cdot 7$ & $1483 \cdot 9-2459 \cdot 6$ & $2618 \cdot 4$ & $2071.8-3217.5$ \\
\hline $\mathrm{Na}(\mathrm{mg})^{*}$ & 1644.6 & $1135 \cdot 6-2257.9$ & 19988.4 & $1353 \cdot 9-2868 \cdot 6$ & 2375.5 & $1636 \cdot 8-3338 \cdot 7$ & $2159 \cdot 1$ & $1466 \cdot 3-3069 \cdot 5$ \\
\hline \multicolumn{9}{|l|}{$\mathrm{Zn}(\mathrm{mg}) \dagger$} \\
\hline Mean & $14 \cdot 1$ & & $12 \cdot 6$ & & $14 \cdot 2$ & & 13.5 & \\
\hline SD & $7 \cdot 3$ & & 6.7 & & 6.8 & & 6.9 & \\
\hline $\mathrm{Cu}(\mathrm{mg})^{\star}$ & 1.6 & $1 \cdot 2-2 \cdot 2$ & 1.4 & $1 \cdot 1-1 \cdot 8$ & 1.5 & $1.2-1.9$ & 1.5 & $1 \cdot 1-2 \cdot 1$ \\
\hline \multicolumn{9}{|l|}{$\mathrm{Mn}(\mathrm{mg}) \dagger$} \\
\hline Mean & $2426 \cdot 8$ & & $2168 \cdot 2$ & & $2358 \cdot 7$ & & $2266 \cdot 2$ & \\
\hline SD & $1537 \cdot 1$ & & $1143 \cdot 7$ & & $1000 \cdot 5$ & & 1204.8 & \\
\hline Vitamin $A(\mu \mathrm{g})^{\star}$ & $1744 \cdot 2$ & $1158 \cdot 1-2536 \cdot 3$ & 1393.3 & $858 \cdot 8-2122 \cdot 8$ & 133.0 & $889 \cdot 2-2073 \cdot 2$ & 1638.1 & $1058 \cdot 6-2314 \cdot 1$ \\
\hline Thiamine $(\mathrm{mg})^{*}$ & $2 \cdot 0$ & $1.6-2.5$ & 1.5 & $1 \cdot 2-2 \cdot 0$ & 1.8 & $1 \cdot 4-2 \cdot 3$ & 1.9 & $1.4-2.5$ \\
\hline Riboflavin $(\mathrm{mg})^{\star}$ & 1.7 & $1 \cdot 2-2 \cdot 6$ & 1.6 & $1 \cdot 2-2 \cdot 1$ & 1.6 & $1 \cdot 2-2 \cdot 3$ & 1.6 & $1 \cdot 1-2.5$ \\
\hline $\operatorname{Niacin}(m g)^{*}$ & $26 \cdot 0$ & $19 \cdot 1-36 \cdot 4$ & $21 \cdot 7$ & $16 \cdot 0-28 \cdot 6$ & 23.7 & $18 \cdot 1-30 \cdot 6$ & 25.6 & $21 \cdot 1-32 \cdot 5$ \\
\hline Vitamin B6 $(\mu \mathrm{g})^{\star}$ & 3.8 & $2 \cdot 8-5 \cdot 3$ & 3.3 & $2 \cdot 2-4 \cdot 2$ & 2.8 & $2 \cdot 0-3 \cdot 8$ & 4.1 & $3 \cdot 2-4 \cdot 9$ \\
\hline Folate $(\mu \mathrm{g})^{\star}$ & $500 \cdot 7$ & $355 \cdot 4-674 \cdot 1$ & 436.6 & $334.8-604.9$ & 448.5 & $343.9-578 \cdot 3$ & $481 \cdot 4$ & $374 \cdot 6-612 \cdot 2$ \\
\hline Vitamin $B_{12}(\mu \mathrm{g})$ * & $5 \cdot 0$ & $2.9-8.5$ & 4.5 & $2 \cdot 5-7 \cdot 3$ & 4.5 & $2 \cdot 7-6 \cdot 9$ & 4.9 & $2.7-7.9$ \\
\hline Pantothenic acid $(\mathrm{mg})^{\star}$ & 5.7 & $4 \cdot 4-7 \cdot 3$ & $5 \cdot 0$ & $3.7-6.4$ & $5 \cdot 3$ & $3 \cdot 9-6 \cdot 7$ & $5 \cdot 8$ & $3.9-8.1$ \\
\hline Biotin $(\mu \mathrm{g})^{\star}$ & 53.7 & $35 \cdot 5-75 \cdot 7$ & 46.9 & $33 \cdot 2-65 \cdot 6$ & $47 \cdot 2$ & $34.7-62 \cdot 1$ & 49.9 & $37 \cdot 4-7$ \\
\hline Vitamin C $(\mathrm{mg})^{*}$ & 79.0 & $45 \cdot 3-153 \cdot 6$ & 41.9 & $28 \cdot 4-78 \cdot 1$ & 51.8 & $29.7-94 \cdot 1$ & 55.5 & $34 \cdot 3-94 \cdot 1$ \\
\hline Vitamin D $(\mathrm{mg})^{*}$ & 4.0 & $2 \cdot 2-6 \cdot 9$ & 3.8 & $2 \cdot 2-6 \cdot 5$ & 4.4 & $2 \cdot 6-6 \cdot 9$ & 4.8 & $2.9-6.9$ \\
\hline Vitamin $E(\mathrm{mg})^{*}$ & 14.1 & $9 \cdot 1-19 \cdot 1$ & 11.7 & $8 \cdot 1-17 \cdot 8$ & $13 \cdot 2$ & $9 \cdot 3-18 \cdot 9$ & 13.9 & $9.2-19.5$ \\
\hline
\end{tabular}

CHO; carbohydrate; n/a, not applicable.

* Non-parametric data presented as median (25th-75th percentiles).

P value for significance of differences in nutrient value between each dietary pattern, comparing the highest tertile of each dietary pattern (Wilcoxon signed-rank test for non-parametric data and Paired $t$ test for parametric data). Calculated as

percentages, using each macro-nutrient's energy (kJ/d), divided by total energy from total protein + carbohydrate + fat. 
Brazil (21.5\%), Mexico (29.8\%) and Chile (28.6\%) ${ }^{(10,11,15,16,36,37,44)}$. This indicates that this urban female population of Soweto, South Africa, an upper middle-income country, has higher percentages of ultra-processed food contributions in their diets than other middleincome countries and similar percentage intakes than high-income countries. However, cultural influences and preferences of different countries may also influence the amount of ultra-processed food consumed.

The high percentage ultra-processed food contributions to total EI in this study reflect the changes in dietary patterns due to the growing obesogenic food environment in South Africa ${ }^{(5,45)}$. In the South African context, cost and affordability can also be a major driver of food choices, irrespective of their nutritional value and quality ${ }^{(46)}$. For example, mass-produced fortified white-and-brown bread, an affordable staple food in South Africa, was the most consumed ultra-processed food item in both breast cancer cases and controls. Compared with affordable unprocessed/minimally processed starchy staples such as rice and maize meal in South Africa, mass-produced white-andbrown breads are cheaper and require less preparation time and may therefore be more frequently bought ${ }^{(47)}$.

Compared with the other NOVA food groups, the ultra-processed food group in our study had the highest content of saturated fat and added sugar while having the lowest content of dietary fibre. A diet consisting mainly of foods with a less healthy nutrient profile (high energy density and high content of added sugar, saturated fat and low intakes of fibre) reduces the overall quality of the diet and increases the risk of developing obesity and other diet-related non-communicable diseases ${ }^{(12-14,40,41,46)}$. The high percentage of ultra-processed food consumption in this study is therefore concerning and emphasises the need for healthy, affordable and sustainable food environments that prioritise nutrition policies supporting the NOURISHING framework $^{(47,48)}$. However, creating healthier and affordable food environments remains a difficult task and will require commitment from various stakeholders across multiple (public, private and individual) sectors in South Africa ${ }^{(49)}$.

Interestingly, no significant associations were observed between higher ultra-processed food consumption and breast cancer risk in this study. In contrast to our findings, the French NutriNet-Santé cohort study (104 980 participants) showed that higher consumption of ultra-processed foods, using the NOVA system, was positively associated with breast cancer risk $^{(10)}$. Another matched case-control study conducted in Brazil found that regular consumption of ultra-processed foods $(>5$ portions per week) was associated with having a 2.35 times higher odds of developing breast cancer ${ }^{(11)}$. Although the ultra-processed food group in our study had a high content of energy, saturated fat and added sugar, together with a low content of dietary fibre, a high content of micronutrients was observed. The high content of micronutrients, which may increase the quality of the overall diet, may explain why no significant association between ultra-processed food consumption and breast cancer risk was observed in our study. It is therefore possible that the type and nutritional value of ultra-processed foods consumed in South Africa differ from the French and Brazilian studies. However, ultra-processed foods are not considered healthy since evidence has linked higher consumption thereof to various non-communicable diseases and therefore requires more research.

Given that cancer develops over an extended period, diet in the past several years may also influence cancer risk. Therefore, results of our study should be interpreted with caution since data collection of the SABC study focused on the participant's current diet (past month at the time of diagnosis) and not past dietary intake of several years back. However, this is a general problem for any study that collect current dietary data only once. Additionally, it is possible that the period of data collection in our study overlapped with breast cancer symptoms, which could have led to changes in habitual dietary intake of breast cancer cases. Thus, a possibility of biased dietary data for cases due to changes in dietary intake, but dietary intake may also depend on the stage of cancer at diagnoses and therefore, require more research.

Our study has some strengths and limitations. Strengths include that cases were recruited prior to any breast cancer treatment and questionnaires used to obtain data were proven to be validated. Trained personnel were used to administer all questionnaires (not self-reported). Data used in the analysis were standardised and the categorisation of the food items according to the NOVA system was discussed with experts involved in the development of the NOVA system. Limitations include the relatively small sample size of this study, especially for subgroup analyses, and the fact that dietary intake was measured over the past month (at recruitment of the study) when habitual dietary intake of case participants could have changed due to illness (increased possibility for reverse causation, but may be dependent on the tumour size and requires more research). The nature of the study design could potentially result in non-differential misclassification and underestimation of the associations. In addition, although dietary intakes were captured throughout the year (in different participants) seasonal variability of foods (not adjusted for) may have influenced usual reporting of dietary intakes. The QFFQ used to evaluate the NOVA food groups was not specifically designed to capture foods based on their degree of processing. This made the recognition of each NOVA food group difficult. In addition, foods were categorised into their most usual form of consumption with the most conservative classification option (less processed level) chosen in a case of doubt. Information bias may therefore exist, while residual confounding can also not be ruled out.

In conclusion, unprocessed/minimally processed foods may reduce the risk of developing breast cancer in this population. Foods associated with unprocessed/minimally processed foods may play an important role in primary breast cancer prevention guidelines. However, more research on this topic is required and our results first need to be replicated in other South African regions and populations before any dietary guidelines can be formulated. Our results suggest that food environments and dietary intake behaviour should be prioritised to make unprocessed/minimally processed foods more affordable, accessible and available in South Africa. The high amount of EI from ultra-processed foods is worrisome as these foods may displace possibly protective foods from the unprocessed $/ \mathrm{minimally}$ processed food group, which may reduce the risk of breast cancer in this population. 


\section{Supplementary material}

For supplementary material accompanying this paper visit https://doi.org/10.1017/S0007114522000423

\section{Acknowledgements}

The authors would like to thank the participants of the SABC study and the Directors of the Chris Hani Baragwanath Academic Hospital and Chris Hani Baragwanath Breast Unit. The authors would also like to thank the fieldworkers involved in this study: Phindile Mathe, Yvonne Chaka, Victor Shandukani, Siphesihle Sibiya and Maria Sihlo and the SABC study project coordinators from the International Agency for Research on Cancer, Tracy Lignini and Robyn Smith. We also acknowledge the contribution towards dietary data collection, coding of QFFQ's and scientific input of prof H.H. Vorster (posthumous) in this study.

This study was supported by a research grant secured from the WCRF, international grant number 2012/591. The WCRF had no role in the study design, analysis or writing of this article.

The authors' contributions were as follows: I. J. formal statistical analysis and writing original draft; C. T.-K. writing - reviewing and editing, involved in conceptualisation, investigation and supervision of the study; M. W. writing - reviewing and editing; H. C. South African principle investigator of SABC study, COresponsible for methodology and resources; M. J. involved in the South African project administration of the SABC study; R. L. formal analysis of dietary intake; I. R. and S. R. head principal investigator of SABC, writing - reviewing and editing; C. B. oversaw formal statistical analysis; R. B. L. writing - reviewing and editing; F. R. writing - reviewing and editing, I. H. writing review and editing.

The authors declare that there are no conflicts of interest.

\section{References}

1. Ferlay J, Ervik M, Lam F, et al. (2020) Global Cancer Observatory: Cancer Today. Lyon, France: International Agency for Research on Cancer.

2. National Institute for Communicable Diseases (2017) Cancer in South Africa 2017 Full Report: National Cancer Registry. https:// www.nicd.ac.za/centres/national-cancer-registry/ (accessed July 2021).

3. World Cancer Research Fund \& American Institute for Cancer Research (2018) Diet, Nutrition, Physical Activity and Cancer: a Global Perspective. Continuous Update Project Expert Report 2018. https://www.dietandcancerreport.org (accessed March 2020).

4. Hawkes C, Jewell J \& Allen K (2013) A food policy package for healthy diets and the prevention of obesity and diet-related non-communicable diseases: the NOURISHING framework. Obes Rev 14, 159-168.

5. Ndlovu N, Day C, Aagaard-Hansen J, et al. (2018) Assessment of food environments in obesity reduction: a tool for public health action. S Afr Health Rev 1, 115-123.

6. Kroll F, Swart EC, Annan RA, et al. (2019) Mapping obesogenic food environments in South Africa and Ghana: correlations and contradictions. Sustainability 11, 3924.
7. Monteiro CA, Cannon G, Lawrence M, et al. (2019) UltraProcessed Foods, Diet Quality, and Health using the NOVA Classification System. Rome: FAO.

8. Baker P, Machado P, Santos T, et al. (2020) Ultra-processed foods and the nutrition transition: global, regional and national trends, food systems transformations and political economy drivers. Obes Rev 21, e13126.

9. Igumbor EU, Sanders D, Puoane TR, et al. (2012) 'Big food,' the consumer food environment, health, and the policy response in South Africa. PLoS Med 9, e1001253.

10. Fiolet T, Srour B, Sellem L, et al. (2018) Consumption of ultraprocessed foods and cancer risk : results from NutriNet-Santé prospective cohort. BMJ 1, 360-322.

11. Queiroz SA, de Sousa IM, de Melo Silva FR, et al. (2018) Nutritional and environmental risk factors for breast cancer: a case-control study. Sci Med 28, 2.

12. Rauber F, Chang K, Vamos EP, et al. (2021) Ultra-processed food consumption and risk of obesity: a prospective cohort study of UK Biobank. Eur J Nutr 60, 2169-2180.

13. Pagliai G, Dinu M, Madarena MP, et al. (2018) Consumption of ultra-processed foods and health status: a systematic review and meta-analysis. Br J Nutr 125, 308-318.

14. Askari M, Heshmati J, Shahinfar H, et al. (2020) Ultra-processed food and the risk of overweight and obesity: a systematic review and meta-analysis of observational studies. Int J Obes 44, 2080-2091.

15. Machado PP, Steele EM, Levy RB, et al. (2019) Ultra-processed foods and recommended intake levels of nutrients linked to non-communicable diseases in Australia: evidence from a nationally representative cross-sectional study. BMJ Open $\mathbf{9}$, e029544.

16. Marrón-Ponce JA, Flores M, Cediel G, et al. (2019) Associations between consumption of ultra-processed foods and intake of nutrients related to chronic non-communicable diseases in Mexico. J Acad Nutr Diet 119, 1852-1865.

17. Eicher-Miller HA, Fulgoni VL \& Keast DR (2012) Contributions of processed foods to dietary intake in the US from 2003-2008: a report of the Food and Nutrition Science Solutions Joint Task Force of the Academy of Nutrition and Dietetics, American Society for Nutrition, Institute of Food Technologists, and International Food Information Council. J Nutr 142, 2065S$2072 S$.

18. Poti JM, Mendez MA, Ng SW, et al. (2015) Is the degree of food processing and convenience linked with the nutritional quality of foods purchased by US households? Am J Clin Nutr 101, $1251-1262$

19. Slimani N, Deharveng G, Southgate DAT, et al. (2009) Contribution of highly industrially processed foods to the nutrient intakes and patterns of middle-aged populations in the European Prospective Investigation into Cancer and Nutrition study. Eur J Clin Nutr 63, S206-S225.

20. Ireland JD \& Møller A (2010) LanguaL food description: a learning process. Eur J Clin Nutr 64, S44-S48.

21. Santos FS, Dias MD, Mintem GC, et al. (2020) Food processing and cardiometabolic risk factors: a systematic review. Rev Saude Publica 54, 70.

22. Monteiro CA, Cannon G, Levy RB, et al. (2019) Ultra-processed foods: what they are and how to identify them. Public Health Nutr 22, 936-941.

23. Jacobs I, Taljaard-Krugell C, Wicks M, et al. (2021) Dietary patterns and breast cancer risk in Black Urban South African Women: the SABC Study. Nutrients 13, 4106.

24. Allred DC, Clark GM, Elledge R, et al. (1993) Association of p53 protein expression with tumor cell proliferation rate and clinical outcome in node-negative breast cancer. J Natl Cancer Inst $\mathbf{8 5}$, 200-206. 
25. Romieu I, Biessy C, Joffe M, et al. (2021) Reproductive factors and risk of breast cancer in black South African women. Cancer Causes Control 32, 415-422.

26. Jacobs I, Taljaard-Krugell C, Ricci C, et al. (2019) Dietary intake and breast cancer risk in black South African women: the South African Breast Cancer study. BrJ Nutr 121, 591-600.

27. Dupont WD \& Plummer WD (1998) Power and sample size calculations for studies involving linear regression. Control Clin Trial 19, 589-560.

28. Ainsworth BE, Haskell WL, Whitt MC, et al. (2000) Compendium of physical activities: an update of activity codes and MET intensities. Med Sci Sports Exerc 32, S498-S504.

29. Romieu I \& Lajous M (2009) The role of obesity, physical activity and dietary factors on the risk for breast cancer: Mexican experience. Salud Pública Méx 51, s172-s180.

30. Vorster HH, Venter CS, Wissing MP, et al. (2005) The nutrition and health transition in the North West Province of South Africa: a review of the THUSA (Transition and Health during Urbanisation of South Africans) study. Public Health Nutr 8, 480-490.

31. MacIntyre U, Venter C \& Vorster HH (2001) A culture-sensitive quantitative food frequency questionnaire used in an African population: 1. Development and reproducibility. Public Health Nutr 4, 53-62.

32. Venter C, MacIntyre U \& Vorster HH (2000) The development and testing of a food portion photograph book for use in an African population. J Hum Nutr Diet 13, 205-218.

33. Wentzel-Viljoen E, Laubscher R \& Kruger A (2011) Using different approaches to assess the reproducibility of a culturally sensitive quantified food frequency questionnaire. SAfrJClin Nutr 24, 143-148.

34. Jacobs I, Taljaard-Krugell C, Wicks M, et al. (2021) Adherence to cancer prevention recommendations is associated with a lower breast cancer risk in black urban South African women. BrJ Nutr 1, 1-12.

35. Wolmarans P, Danster N, Dalton A, et al. (2010) Condensed Food Composition Tables for South Africa. Parrow Valley, South Africa: Medical Research Council.

36. Cediel G, Reyes M, da Costa Louzada ML, et al. (2018) Ultraprocessed foods and added sugars in the Chilean diet. Public Health Nutr 21, 125-133.

37. Moubarac J-C, Batal M, Louzada M, et al. (2017) Consumption of ultra-processed foods predicts diet quality in Canada. Appetite 108, 512-520.
38. Xiao Y, Xia J, Li L, et al. (2019) Associations between dietary patterns and the risk of breast cancer: a systematic review and meta-analysis of observational studies. Breast Cancer Res 21, 16.

39. Dandamudi A, Tommie J, Nommsen-Rivers L, et al. (2018) Dietary patterns and breast cancer risk: a systematic review. Anticancer Res 38, 3209-3222.

40. Vorster HH, Badham J \& Venter C (2013) An introduction to the revised food-based dietary guidelines for South Africa. $S$ Afr J Clin Nutr 26, S5-S12.

41. Rauber F, da Costa Louzada ML, Steele EM, et al. (2018) Ultraprocessed food consumption and chronic non-communicable diseases-related dietary nutrient profile in the UK (2008-2014). Nutrients 10, 587.

42. Juul F, dos Santos Simões B, Litvak J, et al. (2019) Processing level and diet quality of the US grocery cart: is there an association? Public Health Nutr 22, 2357-2366.

43. Martínez Steele E, Baraldi LG, Louzada ML, et al. (2016) Ultraprocessed foods and added sugars in the US diet: evidence from a nationally representative cross-sectional study. BMJ $\mathbf{6}$, e009892.

44. Vandevijvere S \& De Ridder K (2018) Consumption of ultraprocessed food products and diet quality among Belgian children and adults. Eur J Public Health 28, cky213.679.

45. Moodie R, Bennett E, Kwong EJL, et al. (2021) Ultra-processed profits: the political economy of countering the global spread of ultra-processed foods - a synthesis review on the market and political practices of transnational food corporations and strategic public health responses. Int J Health Policy Manag (In the Press).

46. Koen N, Wentzel-Viljoen E \& Blaauw R (2018) Price rather than nutrition information the main influencer of consumer food purchasing behaviour in South Africa: a qualitative study. Int J Consum Stud 42, 409-418.

47. NAMC (2021) Food Basket Price Monthly. http://www.namc. co.za/wp-content/uploads/2021/01/Food-Basket-January2020.pdf (accessed May 2021).

48. Hawkes C (2018) Chapter nine. Globalization and the nutrition transition: a case study (10-1). In Case Studies in Food Policy for Developing Countries: Institutions and International Trade Policies, pp. 113-128 [P Pinstrup-Andersen \& F Cheng, editors]. New York: Cornell University Press.

49. Spires M, Delobelle P \& Sanders D (2016) Diet-related non-communicable diseases in South Africa: determinants and policy responses. S Afr Health Rev 1, 35-42. 